Preprint typeset in JHEP style - PAPER VERSION

UOC-TP 012/10, YITP-10-48, arXiv:1006.4495

\title{
Warped compactification on curved manifolds
}

\author{
Ishwaree P. Neupane \\ Department of Physics and Astronomy, University of Canterbury \\ Private Bag 4800, Christchurch 8020, New Zealand \\ and \\ Yukawa Institute for Theoretical Physics, Kyoto University \\ Kyoto 606-8502, Japan \\ E-mail:ishwaree.neupane@canterbury.ac.nz
}

\begin{abstract}
The characterization of a six- (or seven)-dimensional internal manifold with metric as having positive, zero or negative curvature is expected to be an important aspect of warped compactifications in supergravity. In this context, Douglas and Kallosh recently pointed out that a compact internal space with negative curvature could help to construct four-dimensional de Sitter solutions only if the extra dimensions are strongly warped or there are large stringy corrections. That is, the problem of finding 4-dimensional de Sitter solutions is well posed, if all extra dimensions are physically compact, which is called a no-go theorem. Here, we show that the above conclusion does not extend to a general class of warped compactifications in classical supergravity that allow a non-compact direction or cosmological solutions for which the internal space is asymptotic to a cone over a product of compact Einstein spaces or spheres. For clarity, we present classical solutions that compactify higher-dimensional spacetime to produce a Robertson-Walker universe with de Sitter-type expansion plus one extra non-compact direction. Such models are found to admit both an effective four-dimensional Newton constant that remains finite and a normalizable zero-mode graviton wavefunction. We also exhibit the possibility of obtaining $4 \mathrm{D}$ de Sitter solutions by including the effect of fluxes ( $\mathrm{p}$-form field strengths).
\end{abstract}

KEYWORDS: de Sitter solutions, warped extra dimensions, supergravity. 


\section{Introduction}

Since the beginning of the 20th century, physicists have struggled to find a way to unite general relativity and quantum mechanics. One of the methods by which theoretical physicists have tried to unify gravity with the other forces is to build models that allow for many more spatial dimensions. A strong motivation for considering additional dimensions of space came from string and supergravity theories, which in turn are inspired by the failure of classical gravity to work at very short distance scales.

Given that our universe is described by fundamental theories of higher-dimensional gravity, such as, string/M theory, there should exist six or seven extra dimensions of space, not yet detected by experiment. This is possible if the extra dimensions take the form of a small compact manifold $\mathcal{M}$ or are strongly warped along the transverse direction, giving rise to a finite warped volume for these extra spaces.

In recent years, much of the focus has been on the study of $(4+m)$-dimensional supergravity theories defined on non-factorizable background spacetimes, instead of on the conventional Kaluza-Klein (or product-space) compactifications, $M^{m+4} \rightarrow M^{4} \times M^{m}$. An explicit example of this category is the five-dimensional braneworld model studied by Randall and Sundrum [1], which has brought new prospective in our thinking about the role of gravity in higher dimensions, providing an alternative to the standard Kaluza-Klein or product-space compactification. The RS single brane model [2] has further raised the possibility that, at least, one of the extra dimensions postulated by string/M theory could be large enough to have some new cosmological and phenomenological implications [3].

The 5D braneworld construction is sufficiently simple at a level of model building in particle physics, but it is incomplete in many ways. On general grounds, there is no strong reason to consider just one extra dimension, instead of six or seven as predicted by string or $\mathrm{M}$ theory. In the last ten years or so, a lot of work has been done in this direction, taking into account the effect of background fluxes (form fields) and D-branes. The existence of branes, fluxes and localized objects like an orientifold plane in some fundamental theories of gravity, including string theory, has benefited physicists to develop new ideas, including the localization of gravity on D3-branes, dualities between closed string theories that contain gravity and decoupled open string (or gauge) theories $[4,5]$ and methods of constructing metastable de Sitter vacua using warped extra dimensions [6].

Despite several novelties of string/M theory, including microscopic descriptions of inflation from D-braneworld models [7-11], it is not straightforward to explain an accelerated expansion of the universe directly through compactification of supergravity models in 10 or 11 dimensions (which are the low energy limits of string theory and M-theory). Specifically, there is a 'no-go theorem', due to Gibbons [12], De Wit et al [13], Maldacena and Nunez [14], Giddings et al [15] and many others, which basically asserts that if we dimensionally compactify any string-derived supergravity model on a smooth compact internal manifold $\mathcal{M}$, then we often end up with a flat Minkowski space as a viable background solution of classical supergravities, unless we violate certain positivity conditions. The problem of finding de Sitter solutions is well posed, if all extra dimensions are physically compact. Since the universe is evidently both past and future de Sitter (albeit with vastly differing vacuum energies) this would seem to be a problem.

In recent years, attempts have been made around this particular 'no-go' result. The original "no-go" theorem assumes time independence of the internal space, and so one could look for time-dependent solutions. Following this intuition, Chen et al [16], Townsend and 
Wohlfarth [17], Ohta [18], Neupane et al [19] and others [15] constructed time-dependent solutions of ten and eleven-dimensional supergravities which describe one or more periods of transient acceleration of the universe; we refer to $[20,21]$ for further references. The basic idea is simple: given that the internal space is described by certain metric moduli associated with the internal space scale factors and these moduli describe the size and other basic properties of the internal manifold, then upon dimensional reduction, time-dependent metric moduli typically give rise to an exponential potential in lower dimensions [22]. In the case of pure $D$-dimensional gravity $[19,20]$, one would require one or more internal subspaces to have negative curvature, particularly, with time-dependent metric moduli, which generate a sum of positive exponential potentials in lower dimensions. The background fluxes provide an additional contribution to them. One can now imagine 'bouncing' the universe off an effective potential [23]. Albeit for a brief interval, the energy is dominated by the potential term, and the universe undergoes a transient period of cosmic acceleration. All higher dimensional models with time-dependent metric moduli field inherit the property that as one moves to the minimum of an effective potential the size of extra dimensions grows only slowly or even stabilise in a few specific cases [24].

One particular reason to be interested in warped geometries (with an internal space of arbitrary curvature) is to find 4D de Sitter solutions. To this end, one finds natural interest in supergravity models, where the extra dimensions are warped but time-independent. In this context, Douglas and Kallosh recently pointed out that a compact internal space with negative curvature could help us to construct four-dimensional de Sitter solutions only if the extra dimensions are strongly warped or there are large stringy corrections [25]. That is, the problem of finding 4-dimensional de Sitter solutions is well posed, if all extra dimensions are physically compact, which is called a no-go theorem. In this paper, we show that within a certain class of warped supergravity models it won't be necessary to introduce stringy corrections and/or some localized brane sources violating certain positivity conditions just for the purpose of getting $4 \mathrm{D}$ de Sitter solutions. Here we give some explicit examples for which the $4 \mathrm{D}$ effective Planck mass is finite, despite having a noncompact direction, and the $4 \mathrm{D}$ de Sitter solutions exist with an arbitrary choice of the internal curvature.

The earlier results, for instance, in $[5,15]$ show that, in a non-cosmological setting, a nontrivial warp factor could arise as the backreaction of some (background) fluxes. However, in a cosmological setting, in which one replaces a flat 3-brane by some cosmologically relevant metrics, such as, FLRW universe, we find that a non-trivial warp factor could by itself give rise to de Sitter solutions in four dimensions. We show that one can obtain a de Sitter solution even when the internal manifold is Ricci flat, provided that the warp factors for both the external $4 \mathrm{D}$ spacetime and the internal 6D space are nontrivial. This work significantly extends and generalizes the earlier analysis in [26].

Some of the examples of solutions presented in this paper may look closer in spirit to Randall-Sundrum-type braneworld constructions [27]. The important difference here is that the effect of a compact base manifold $X_{5}$ (or $X_{6}$ in the $D=11$ case) is also incorporated in the classical solutions. Our construction is motivated from 10D and 11D supergravity theories, in which one takes into account the effect of $p$-form gauge fields.

The paper is organized as follows: In section 2, we start with a warped $10 \mathrm{D}$ metric ansatz, which is the product of a maximally symmetric $4 \mathrm{D}$ spacetime and a general $6 \mathrm{D}$ Einstein space of arbitrary curvature. We solve the 10D Einstein equations without fluxes (or source terms) and show that they lead to de Sitter solutions in four dimensions. Our examples lead to a finite warped volume and hence a finite 4D effective Planck mass. 
In section 3, we briefly discuss on why the 'warping' of extra spaces might play an important role in the construction of de Sitter solutions, generalizing earlier works in $[26$, 28]. We also discuss the differences and advantages of adding energy sources for p-branes and fluxes. In section 4, we analyze 10D supergravity flux equations. We use our knowledge of the background de Sitter solutions to explore the possibility of having a negatively curved $6 \mathrm{D}$ space but again without introducing brane sources or objects violating 'internal energy' conditions. The addition of $p$-form fluxes does not much affect the nature of $4 \mathrm{D}$ de Sitter solutions except in the limit where the base manifold $X_{5}$ shrinks to zero size. In section 5, we present a four-dimensional de Sitter solution by solving Einstein's equations in 11 dimensions. In section 6 , we briefly summarize our results.

\section{Warped metrics}

We begin with a ten-dimensional metric ansatz of the form

$$
d s_{10}^{2}=\tau^{2} e^{\beta A(y)} \hat{g}_{\mu \nu} d x^{\mu} d x^{\nu}+\rho^{2} e^{\alpha A(y)} d s_{6}^{2},
$$

with

$$
d s_{6}^{2}=g_{i j}^{(6)}(y) d y^{i} d y^{j},
$$

where $\beta, \alpha$ are numerical constants, and $\tau^{2}$ and $\rho^{2}$ are some other constants which can be related to extremized values of $10 \mathrm{D}$ dilaton and volume modulus. For the metric (2.1), with $X_{6}$ as a compact internal space, we find

$$
\begin{gathered}
{ }^{(10)} R_{\mu \nu}(x, y)={ }^{(4)} \hat{R}_{\mu \nu}(x)-\frac{\tau^{2}}{\rho^{2}}\left(\beta^{2}+\beta \alpha\right)(\nabla A)^{2} e^{(\beta-\alpha) A(y)} \hat{g}_{\mu \nu}-\frac{\tau^{2}}{\rho^{2}} \frac{\beta}{2} e^{(\beta-\alpha) A(y)} \hat{g}_{\mu \nu} \nabla_{y}^{2} A \\
{ }^{(10)} R_{i j}(x, y)= \\
{ }^{(6)} \tilde{R}_{i j}-\left(\alpha^{2}+\beta \alpha\right)(\nabla A)^{2} \tilde{g}_{i j}^{(6)}-2(\beta+\alpha) \nabla_{i} \nabla_{j} A \\
-\left(\beta^{2}-2 \beta \alpha-\alpha^{2}\right) \nabla_{i} A \nabla_{j} A-\frac{\alpha}{2} \tilde{g}_{i j}^{(6)} \nabla_{y}^{2} A
\end{gathered}
$$

This result corrects a couple of typos/errors in [29]. In the above $\tilde{g}_{i j}^{(6)}$ denote the metric components of the internal space, which are independent of the $y$ coordinate. The notations here follow that from refs $[26,28]$.

\subsection{A canonical choice of $6 \mathrm{D}$ metric}

The simplest examples of $6 \mathrm{D}$ compact spaces are given by ${ }^{1}$

$$
\begin{aligned}
& d s_{6}^{2}=d y^{2}+d y_{1}^{2}+\cdots+d y_{5}^{2}, \quad(\epsilon=0) \\
& d s_{6}^{2}=d y^{2}+\sin ^{2} y d \Omega_{5}^{2}, \quad(\epsilon=+1) \\
& d s_{6}^{2}=d y^{2}+\sinh ^{2} y d \Omega_{5}^{2}, \quad(\epsilon=-1),
\end{aligned}
$$

where $d \Omega_{5}^{2}$ represents the metric of a 5 -sphere, ${ }^{(6)} \tilde{R}_{i j}=\epsilon(m-1) \tilde{g}_{i j}$, with $m$ being the number of extra dimensions and $m=6$ in this example. Furthermore,

$$
\nabla_{y}^{2} A= \begin{cases}A^{\prime \prime}, & (\epsilon=0) \\ A^{\prime \prime}+5 A^{\prime} \cot y, & (\epsilon=+1) \\ A^{\prime \prime}+5 A^{\prime} \operatorname{coth} y, & (\epsilon=-1)\end{cases}
$$

\footnotetext{
${ }^{1}$ In the $\epsilon=-1$ case, a compact hyperbolic manifold is achieved by taking a quotient $H^{6} /\left.\Gamma\right|_{\text {free }}$ of the non-compact space $H^{6}$ by a freely acting discrete subgroup $\Gamma$ of the isometry group.
} 
Let us first consider a specific example, with $\alpha=0$ and $\beta=2$. For simplicity, we set $\tau=\rho=1$. Equations (2.3) and (2.4) reduce to ${ }^{2}$

$$
{ }^{(10)} R_{\mu}^{\mu}=e^{-2 A} \hat{R}_{4}-16\left(\nabla_{y} A\right)^{2}-4 \nabla_{y}^{2} A,
$$

and

$$
{ }^{(10)} R_{i}^{i}=\tilde{R}_{6}-4 \nabla_{i} \partial^{i} A-4 \nabla_{i} A \partial^{i} A .
$$

Note that, since $A \equiv A(y)$, the last two terms above contribute only when $i=y$.

Now, from the trace-subtracted 10D Einstein equations, i.e.

$$
{ }^{(10)} R_{A B}=T_{A B}-\frac{1}{8} g_{A B} T_{C}^{C},
$$

we obtain

$$
\begin{aligned}
{ }^{(10)} R_{\mu}^{\mu} & =\frac{1}{2} T_{\mu}^{\mu}-\frac{1}{2} T_{m}^{m} \equiv \frac{1}{2} T_{4}-\frac{1}{2} T_{6}, \\
{ }^{(10)} R_{i}^{i} & =\frac{1}{4} T_{i}^{i}-\frac{3}{4} T_{\mu}^{\mu} \equiv \frac{1}{4} T_{6}-\frac{3}{4} T_{4} .
\end{aligned}
$$

To return to the familiar $4 \mathrm{D}$ notations, one may replace $T_{A B}$ by $8 \pi G \mathcal{T}_{A B}$. Here we also introduce the following notations:

$$
\hat{R}_{4}={ }^{(4)} \hat{R}_{\mu}^{\mu}, \quad \tilde{R}_{6}={ }^{(6)} \tilde{R}_{i}^{i}, \quad R^{(6)} \equiv{ }^{(10)} R_{i}^{i} .
$$

In a sense, $R^{(6)}$ is the total integrated $6 \mathrm{D}$ scalar curvature, which is generally positive, while $\tilde{R}_{6}$ is the scalar curvature associated with the $6 \mathrm{D}$ metric itself.

Equating Eqs. (2.7) and (2.8) with Eqs. (2.10) and (2.11), we find

$$
\tilde{R}_{6}=-12\left(\nabla_{y} A\right)^{2}+e^{-2 A} \hat{R}_{4}+\frac{3}{4} T_{6}-\frac{5}{4} T_{4}
$$

Similarly, with $\beta=-\alpha=2$, we find

$$
\tilde{R}_{6}=8\left(\nabla_{y} A\right)^{2}-10 \nabla_{y}^{2} A+e^{-4 A} \hat{R}_{4}+e^{-2 A}\left(\frac{3}{4} T_{6}-\frac{5}{4} T_{4}\right) .
$$

As is evident, different choices of $\beta$ and $\alpha$ can easily lead to different set of equations.

Of course, the above set of equations do not lead to a $4 \mathrm{D}$ de Sitter solution unless that the energy momentum tensor $T_{A B}$ has components that violate certain (positivity) conditions. In a general $D$ dimensions, with $m$ extra dimensions, i.e. $D=d+m$, the strong energy condition is $T_{m} \geq \frac{m-2}{d} T_{d}[12,31]$, which with $d=4$ and $m=6$ reads $T_{6} \geq T_{4}{ }^{3}$.

We have not specified yet the source of energy momentum tensor fields, which may include contributions from a bulk cosmological constant, fluxes or even some non-local effects of warped branes in the extra dimensions, but their explicit expressions won't be important for our discussion in this section. We just want to emphasize here that the condition like $T_{6} \geq T_{4}$ (in $D=10$ dimensions) does not necessarily enforce $\tilde{R}_{6}$ only to take a positive value; the other two choices $\left(\tilde{R}_{6}=0\right.$ and $\left.\tilde{R}_{6}<0\right)$ are also possible.

\footnotetext{
${ }^{2}$ Even though we specified above the metric of the compact $6 \mathrm{D}$ space, the results here are valid for any other 6D spaces, including Kä̈ler manifolds, with an arbitrary curvature. Einstein's equations carry no information about the topology of internal manifold but only its spatial curvature.

${ }^{3}$ In [25], Douglas and Kallosh introduced a new condition, so-called internal energy condition, $T_{m} \geq$ $\frac{m}{d-2} T_{d}$, which can however be violated in $D=10$ by wrapped Dp-branes when $p>7$. This is also a reason for why one considers, for instance, in type IIB string theory, only Dp-branes with $p \leq 7$.
} 


\subsection{A more general choice of $6 \mathrm{D}$ metric}

For the choice of $6 \mathrm{D}$ metrics as in $(2.5 \mathrm{a})-(2.5 \mathrm{c})$, there is no free parameter which may be tuned to the coefficient such as $\alpha$ in the volume factor, which could otherwise lead to a de Sitter solution in four dimensions. If we write the $6 \mathrm{D}$ metric ansatz in the following form:

$$
d s_{6}^{2}=g(y) d y^{2}+\alpha_{1} f(y) d \Omega_{5}^{2},
$$

then we immediately see that a four-dimensional de Sitter solution can be obtained by tuning the coefficient $\alpha$ with the constant $\alpha_{1}$ related to the $6 \mathrm{D}$ curvature. This is possible also for a canonical choice that $g(y)=1$ and $f(y)=y^{2}$. A detailed analysis actually shows that if we set $\alpha=0$ (from the beginning) and allows the $6 \mathrm{D}$ metric to take the form of (2.15), then the solution for the warp factor must take a rather nontrivial form in order to yield de Sitter solutions in four-dimensions. From this we also conclude that even though one could in principle absorb the volume factor $e^{\alpha A(y)}$ within the internal metric (cf equation 2.1) or set $\alpha=0$, there are no advantages with this choice.

\subsection{Nontrivial solutions}

In the analysis below, we write the $6 \mathrm{D}$ metric ansatz in the following form:

$$
d s_{6}^{2}=\sinh ^{2} y d y^{2}+\alpha_{1} \cosh ^{2} y d s_{X_{5}}^{2} .
$$

where $X_{5}$ is either $S^{5}$ or an Einstein-Sasaki space $T^{1,1}=\left(S^{2} \times S^{2}\right) \rtimes S^{1}$. As compared to the metrics $(2.5 \mathrm{a})-(2.5 \mathrm{c})$, we now have one more free parameter, $\alpha_{1}$. The metric $(2.16)$ is Ricci flat only if $\alpha_{1}=1$, while it is positively (negatively) curved for $\alpha_{1}<1\left(\alpha_{1}>1\right)$. There are two motivations behind the above choice of the metric. First, unlike in the examples considered in [30], the size of the compact space $X_{5}$ is nonzero (avoiding a conical singularity at $y=0$ ) and the curvature components are regular everywhere. Second, the problem of finding four-dimensional de Sitter solutions is well posed, especially, if all extra dimensions are physically compact, so we can study higher-dimensional supergravity theories by allowing a noncompact dimension. This approach is perfectly viable and is also consistent with the observation that the Calabi-Yau spaces in string theory are generically noncompact and they are also known to allow at least one noncompact direction, admitting a warped throat geometry.

Of course, using the coordinate transformation $\sinh y d y \rightarrow d z$, we may express (2.16) into a more familiar form $d s_{6}^{2}=d z^{2}+f(z)^{2} d s_{X_{5}}^{2}$, where $f(z) \propto(z+c)$, but we prefer to use the metric (2.16) as it is sufficiently general and also convenient for the purpose of solving Einstein's field equations. With (2.16), we find that the Ricci tensor components of the $10 \mathrm{D}$ spacetime are related to those in $4 \mathrm{D}$ spacetime and the internal spaces by

$$
\begin{array}{r}
{ }^{(10)} R_{\mu \nu}(x, y)={ }^{(4)} \hat{R}_{\mu \nu}(x)-\frac{\hat{g}_{\mu \nu} e^{(\beta-\alpha) A}}{\sinh ^{2} y}\left[\left(\beta^{2}+\beta \alpha\right) A^{\prime 2}+\frac{\beta}{2} A^{\prime \prime}+\frac{\beta}{2}(5 \tanh y-\operatorname{coth} y) A^{\prime}\right], \\
(2.17 \mathrm{a}) \\
R_{y y}=-\frac{4 \beta+5 \alpha}{2} A^{\prime \prime}-\left(\beta^{2}-\beta \alpha\right) A^{\prime 2}+2 \beta A^{\prime} \operatorname{coth} y+\frac{5 \alpha A^{\prime}}{\sinh (2 y)}, \\
(2.17 \mathrm{~b}) \\
{ }^{(10)} R_{p q}=\tilde{R}_{p q}-\tilde{g}_{p q} \alpha_{1} \operatorname{coth}^{2} y\left(\frac{\alpha A^{\prime \prime}}{2}+\left(\alpha \beta+\alpha^{2}\right) A^{\prime 2}+(2 \beta+4 \alpha) A^{\prime} \tanh y-\frac{\alpha A^{\prime}}{\sinh 2 y}\right),
\end{array}
$$


where $^{\prime} \equiv \partial / \partial y$ and

$$
\tilde{R}_{p q} \equiv 4\left(1-\alpha_{1}\right) \tilde{g}_{p q}
$$

Here $\tilde{g}_{p q}$ denote the metric components of the base space $X_{5}$, such as $S^{5}$ or $T^{1,1}$, which are independent of the y coordinate. In the $\alpha \neq \beta$ case, we shall consistently choose

$$
\alpha_{1}=\frac{(\beta-\alpha)^{2}}{2 \beta^{2}}
$$

At this stage, we make no prior assumptions about the internal space curvature.

\subsection{Positive curvature}

Take $\beta=2, \alpha=4$ and $\alpha_{1}=1 / 2$. The internal $6 \mathrm{D}$ space with the metric (2.16) is positively curved, $\tilde{R}_{p q}=2 \tilde{g}_{p q}$. The components of the $10 \mathrm{D}$ Ricci curvature are given by

$$
\begin{gathered}
{ }^{(10)} R_{\mu}^{\mu}={ }^{(4)} \hat{R}_{\mu}^{\mu} e^{-2 A}-\frac{4 e^{-4 A}}{\sinh ^{2} y}\left(12 A^{\prime 2}+\nabla_{y}^{2} A\right)=\frac{1}{2}\left(T_{4}-T_{6}\right), \\
{ }^{(10)} R_{i}^{i}={ }^{(6)} \tilde{R}_{i}^{i} e^{-4 A}-\frac{4 e^{-4 A}}{\sinh ^{2} y}\left(29 A^{\prime 2}+6 \nabla_{y}^{2} A\right)=\frac{1}{4}\left(T_{6}-3 T_{4}\right),
\end{gathered}
$$

where

$$
\tilde{R}_{6}={ }^{(6)} \tilde{R}_{i}^{i}=\frac{20\left(1-\alpha_{1}\right)}{\alpha_{1} \cosh ^{2} y}
$$

and

$$
\nabla_{y}^{2} A=A^{\prime \prime}+(5 \tanh y-\operatorname{coth} y) A^{\prime} .
$$

From equations (2.20a) and (2.20b), one has

$$
\tilde{R}_{6}=\frac{4}{\sinh ^{2} y}\left(17 A^{\prime 2}+5 \nabla_{y}^{2} A\right)+e^{2 A} \hat{R}_{4}+\left(\frac{3}{4} T_{6}-\frac{5}{4} T_{4}\right) e^{4 A} .
$$

Unlike with the maximally symmetric metrics introduced in (2.5a)-(2.5c), which are singular at $y=0$, for the metric (2.16), the $6 \mathrm{D}$ curvature tensors can be regular everywhere.

Here, we take $T_{A B}=0$, so that the condition such as $T_{6} \geq T_{4}$ is trivially satisfied (also saturated), $T_{6}=T_{4}=0$. The $10 \mathrm{D}$ Einstein equations are then explicitly solved when

$$
A(y)=-\ln \cosh y-A_{0}, \quad \hat{R}_{4}=32 e^{2 A_{0}}, \quad \tilde{R}_{6}=\frac{20}{\cosh ^{2} y} .
$$

Note that the warp factor $e^{A(y)} \rightarrow \infty$ when $A_{0} \rightarrow-\infty$, which implies that a flat $4 \mathrm{D}$ Minkowski spacetime is not a solution in the above case. The requirement that the warp factor is real and positive definite also rules out an anti-de Sitter solution. Further, if the $4 \mathrm{D}$ metric takes the form of a standard FRW universe

$$
d s_{4}^{2} \equiv \hat{g}_{\mu \nu} d x^{\mu} d x^{\nu}=-d t^{2}+a^{2}(t)\left[\frac{d r^{2}}{1-k r^{2}}+r^{2} d \Omega_{2}^{2}\right]
$$

where $k$ is the $3 \mathrm{D}$ spatial curvature, then the scale factor is given by

$$
a(t)=\frac{a_{0}}{2} \exp \left(\sqrt{\frac{8}{3}} e^{A_{0}} t\right)+\frac{3 k}{16 a_{0}} e^{-2 A_{0}} \exp \left(-\sqrt{\frac{8}{3}} e^{A_{0}} t\right)
$$


where $a_{0}$ is an arbitrary constant. This is nothing but a de Sitter solution in an accelerating patch ${ }^{4}$. Note that, unlike in pure supergravity theories with a flat 3-brane, for which a nontrivial warp factor only arises as the back-reaction of some fluxes (and hence a nonvanishing $T_{A B}$ ), the vacuum energy on a de Sitter brane (or an inflating FRW universe) can naturally warp the bulk spacetime and introduce a nontrivial warp factor even if $T_{A B}=0$.

\subsection{Zero curvature}

Next, we make the choice $\alpha=(1+\sqrt{2}) \beta$ and $\alpha_{1}=1$. The internal $6 \mathrm{D}$ space with the metric (2.16) is Ricci flat, $\tilde{R}_{p q}=0$. We then find

$$
\begin{array}{r}
\hat{R}_{4} e^{-\beta A}-\frac{e^{-(1+\sqrt{2}) \beta A}}{\sinh ^{2} y}\left((8+4 \sqrt{2}) \beta^{2}(\nabla A)^{2}+2 \beta \nabla^{2} A\right)=\frac{1}{2}\left(T_{4}-T_{6}\right), \\
e^{-(1+\sqrt{2}) \beta A}\left(\tilde{R}_{6}-\frac{1}{\sinh ^{2} y}\left((20+14 \sqrt{2}) \beta^{2}(\nabla A)^{2}+(7+5 \sqrt{2}) \beta \nabla^{2} A\right)\right)=\frac{1}{4}\left(T_{6}-3 T_{4}\right) .
\end{array}
$$

From these equations we derive

$$
\begin{gathered}
\tilde{R}_{6}=\frac{1}{\sinh ^{2} y}\left(\beta^{2}(12+10 \sqrt{2})(\nabla A)^{2}+5(1+\sqrt{2}) \nabla^{2} A\right)+e^{\sqrt{2} \beta A} \hat{R}_{4} \\
+\left(\frac{3}{4} T_{6}-\frac{5}{4} T_{4}\right) e^{(1+\sqrt{2}) \beta A} .
\end{gathered}
$$

With $T_{A B}=0$, the 10D Einstein equations are explicitly solved for

$$
\begin{aligned}
& a(t)=\frac{a_{0}}{2} \exp \left(\sqrt{\frac{4}{3}} e^{A_{0}} t\right)+\frac{3 k}{8 a_{0}} e^{-2 A_{0}} \exp \left(-\sqrt{\frac{4}{3}} e^{A_{0}} t\right), \\
& A(y)=-\frac{1}{\sqrt{2}}\left(\ln \cosh y+A_{0}\right), \quad \tilde{R}_{6}=0 .
\end{aligned}
$$

\subsection{Negative curvature}

Finally, we take $\beta=2, \alpha=6$ and $\alpha_{1}=2$. The internal $6 \mathrm{D}$ space with the metric (2.16) is negatively curved, $\tilde{R}_{p q}=-4 \tilde{g}_{p q}$. We then find

$$
\begin{array}{r}
{ }^{(10)} R_{\mu}^{\mu}={ }^{(4)} \hat{R}_{\mu}^{\mu} e^{-2 A}-\frac{4 e^{-6 A}}{\sinh ^{2} y}\left(16\left(\nabla_{y} A\right)^{2}+4 \nabla_{y}^{2} A\right)=\frac{1}{2}\left(T_{4}-T_{6}\right), \\
{ }^{(10)} R_{i}^{i}={ }^{(6)} \tilde{R}_{i}^{i} e^{-6 A}-\frac{4 e^{-6 A}}{\sinh ^{2} y}\left(58\left(\nabla_{y} A\right)^{2}+\frac{17}{2} \nabla_{y}^{2} A\right)=\frac{1}{4}\left(T_{6}-3 T_{4}\right) .
\end{array}
$$

From these equations we derive

$$
\tilde{R}_{6}=\frac{4}{\sinh ^{2} y}\left(42\left(\nabla_{y} A\right)^{2}+\frac{9}{2} \nabla_{y}^{2} A\right)+e^{4 A} \hat{R}_{4}+\left(\frac{3}{4} T_{6}-\frac{5}{4} T_{4}\right) e^{6 A} .
$$

\footnotetext{
${ }^{4}$ The universe we live in was perhaps not de Sitter at all times. It is easy to understand why this might be the case because the presence of matter fields at later epochs of cosmic evolution can easily lead the expansion away from a pure de Sitter phase. As we discuss below, the consideration of standard background fluxes, or p-form gauge fields, which contribute to $R^{(4)}$ negatively, can also do this job.
} 
With $T_{A B}=0$, the 10D Einstein equations are explicitly solved when

$$
\begin{aligned}
& a(t)=\frac{a_{0}}{2} \exp \left(\sqrt{\frac{2}{3}} e^{A_{0}} t\right)+\frac{3 k}{4 a_{0}} e^{-2 A_{0}} \exp \left(-\sqrt{\frac{2}{3}} e^{A_{0}} t\right), \\
& A(y)=-\frac{1}{2}\left(\ln \cosh y+A_{0}\right), \quad \tilde{R}_{6}=-\frac{10}{\cosh ^{2} y} .
\end{aligned}
$$

Coming to the issue of the Maldacena-Nunez no-go theorem, the main assumption in [14] appeared to be that the internal manifold is physically compact and the integrand $\int \nabla^{2} e^{n A}$ vanishes, where $n$ is some constant. This last condition can be simply relaxed if the extra-dimensional manifold is only geometrically compact or when there exists a noncompact direction (as above). In fact, the quantities such as $\nabla^{2} A$ and $\nabla^{2} e^{n A(y)}$ encode information about the spatial curvature of the internal metric. As a consequence, depending upon the choice of the $6 \mathrm{D}$ curvature, the integrand $\int \nabla^{2} e^{n A}$ may or may not vanish. The other crucial assumption of the MN no-go theorem was that the extra-dimensional warped volume is finite (or a finite Newton's constant), which is however not violated by the solutions presented in this paper.

\subsection{Combining results}

The above results can be expressed in a more illustrative and simpler form:

$$
d s_{10}^{2}=\frac{e^{2 \mathcal{A}}}{(\cosh y+\epsilon \sinh y)^{2 \lambda}}\left[d s_{4}^{2}+\frac{4 L^{2}}{3}\left(2 \lambda^{2}\left(\frac{\epsilon+\tanh y}{1+\epsilon \tanh y}\right)^{2} d y^{2}+d \Omega_{X_{5}}^{2}\right)\right],
$$

where $\lambda$ and $\mathcal{A}$ are dimensionless constants, $L$ has the dimension of length and

$$
d s_{4}^{2}=-d t^{2}+a^{2}(t)\left[\frac{d r^{2}}{1-k r^{2}}+r^{2} d \Omega_{2}^{2}\right], \quad a(t)=\frac{a_{0}^{2}+k L^{2}}{2 a_{0}} \cosh \frac{t}{L}+\frac{a_{0}^{2}-k L^{2}}{2 a_{0}} \sinh \frac{t}{L},
$$

where $a_{0}$, as above, is an arbitrary constant. The metric (2.33) is an exact solution to $10 \mathrm{D}$ Einstein equations without sources $\left(T_{A B}=0\right)$. The solutions presented in the above subsections are obtained by setting $\epsilon=0$, taking a specific value of $\lambda$ and then suitably redefining some of the numerical constants. The above results are new and they are quite remarkable. For the reasons to be explained below we chose $0 \leq \epsilon^{2}<1$ and $\lambda>0$.

As briefly mentioned above, the de Sitter solutions presented here get around the no-go theorem because at least one of the assumptions of the original no-go theorem is violated. Indeed, we have chosen to 'compactify' a string-inspired supergravity model by considering a non-compact dimension. This may sound counterintuitive, but actually it is a well-defined procedure known as consistent warped compactification. To be precise, we will explicitly show that the extra-dimensional warped volume is finite for our solutions and hence the four-dimensional effective Planck mass is also finite.

On passing, we note that the $6 \mathrm{D}$ volume (without the effect of warping) is

$$
V_{6}=\int \sqrt{g_{6}} d^{6} x=\frac{64 \sqrt{2} L^{6}}{27} \operatorname{Vol}\left(X_{5}\right) \int \frac{\epsilon+\tanh y}{1+\epsilon \tanh y} d y
$$

where $\operatorname{Vol}\left(X_{5}\right)=16 \pi^{3} / 27$ if $X_{5}=T^{1,1}$ and $\operatorname{Vol}\left(X_{5}\right)=8 \pi^{2} / 15$ if $X_{5}=S^{5}$. This integral can be explicitly evaluated. We find that

$$
V_{6} \propto(\ln \cosh y+\ln (1+\epsilon \tanh y)) .
$$


We can clearly see the necessity of the choice $\epsilon<1$, especially, in the case y is allowed to range from $-\infty$ to $+\infty$ so that the $6 \mathrm{~d}$ volume is regular everywhere. From the solution (2.33) itself it is clear that, in the large volume limit $y \rightarrow \infty$, the radius modulus, which scales as $|(\epsilon+\tanh y) /(1+\epsilon \tanh y)|$, takes a finite value. Of course, the volume modulus $\sigma \equiv\left(\mathrm{Vol}_{6}\right)^{1 / 3}$ grows linearly with $y^{1 / 3}$, since $|\tanh y| \rightarrow 1$ as $y \rightarrow \infty$. This growth could possibly be halted or minimized, leading to a rough stabilisation of extra dimensional volume, by adding additional energy sources, such as wrapped branes and fluxes, or even some non-perturbative contributions to the vacuum expectation value of $\langle\sigma\rangle$ as in KachruKallosh-Linde-Trevedi (KKLT)-type constructions ${ }^{5}$; we may not even require any such corrections for the purpose of getting a finite warped volume or a finite four-dimensional Newton's constant.

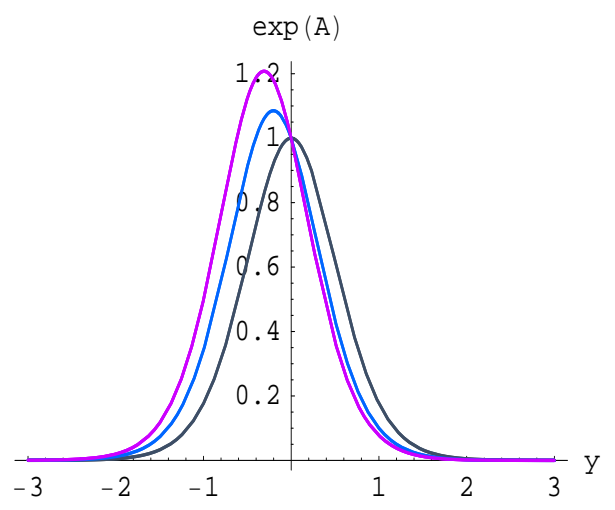

Figure 1: The plot of the warp factor $\exp (A(y))=\exp (\mathcal{A}) /(\cosh y+\epsilon \sinh y)^{\lambda}$ with $\mathcal{A}=0, \lambda=2$ and $\epsilon=0.3,0.2,0$ (from top to bottom) (online: pink, blue and black).

Note that the warp factor

$$
e^{A(y)} \equiv \frac{e^{\mathcal{A}}}{(\cosh y+\epsilon \sinh y)^{\lambda}}
$$

is bounded from the above and below when $\lambda>0$ and $0 \leq \epsilon<1$ (cf figure (1)). Around $y \simeq 0$, we have

$$
e^{2 A} \sim \exp (-2 \lambda|y|) .
$$

As is evident, the larger is the value of $\lambda$ (and hence the longer the throat region), the stronger would be the warping along the transverse direction. For a given $\lambda$ (which we take to be positive) the warped volume would be minimum when $\epsilon=0$. This is more evident from the following explicit result:

$$
\begin{aligned}
\int d^{10} x \sqrt{-g_{10}} R_{10} & =\frac{64 \sqrt{2} L^{6} \lambda}{27} e^{8 \mathcal{A}} \int d \Omega_{5} \int_{y_{0}}^{y_{1}} \frac{\sinh y+\epsilon \cosh y}{(\cosh y+\epsilon \sinh y)^{8 \lambda+1}} d y \int d^{4} x \sqrt{\hat{g}}\left(\hat{R}_{4}-\frac{12}{L^{2}}\right) \\
& =\frac{8 \sqrt{2} L^{6}}{27} e^{8 \mathcal{A}} \operatorname{Vol}\left(X_{5}\right)\left[\frac{-1}{(\cosh y+\epsilon \sinh y)^{8 \lambda}}\right]_{y_{0}}^{y_{1}} \int d^{4} x \sqrt{\hat{g}_{4}}\left(\hat{R}_{4}-\frac{12}{L^{2}}\right) .
\end{aligned}
$$

\footnotetext{
${ }^{5}$ In such a case, the solution to $10 \mathrm{D}$ supergravity equations may not be exactly of de Sitter type, since additional sources or fluxes can modify both the warp/conformal factors and the 4D curvature.
} 
As long as $\epsilon^{2}<1$ and $\lambda>0$, the $6 \mathrm{D}$ warped volume is finite. A large and positive $\lambda$ implies a strong warping of extra spaces and also leads to a relatively large throat region (internal volume) ${ }^{6}$. Let us take $\epsilon=0$, just for simplicity. We then obtain

$$
\frac{M_{(10)}^{8}}{(2 \pi)^{6}} \int d^{10} x \sqrt{-g_{10}} R_{10}=M_{\mathrm{Pl}}^{2} \int d^{4} x \sqrt{-g_{4}}\left(R_{4}-\Lambda_{4}\right),
$$

where $M_{(10)}$ is the fundamental 10D Planck scale, $\Lambda_{4} \equiv 12 / L^{2}$ and

$$
M_{\mathrm{Pl}}^{2}=\frac{M_{(10)}^{8} e^{8 \mathcal{A}}}{(2 \pi)^{6}} V_{6}^{\mathrm{w}}, \quad V_{6}^{\mathrm{w}} \equiv \operatorname{Vol}\left(X_{5}\right) \frac{8 \sqrt{2} L^{6}}{27} \times\left.\frac{2}{\cosh (y)^{8 \lambda}}\right|_{y_{0}} ^{y_{1}} .
$$

Note that one does not have to introduce any artificial cutoff to make the Newton's constant finite in (2.40); the integrand is finite even when we take $y_{0}=-\infty$ and $y_{1}=+\infty$. Of course, one may take $y_{0}=y_{\mathrm{IR}}$ and $y_{1}=y_{\mathrm{UV}}$, without loos of generality. Moreover, the size of the radial (fifth) dimension, along with the size of compact $X_{5}$ manifold, takes a constant value in the large volume limit, providing an explicit example of spontaneous compactification. The stability of the compactified space is guaranteed at least at a classical level. We have also checked that there is a zero-mode graviton wavefunction that is normalizable in the usual $3+1$ spacetime [33]. In a follow up work we will study in detail the effects of continuum KK modes on a 4D gravitational potential and related physics, where we also take into account the effects of a scalar field Lagrangian.

It may be worth pointing out a few crucial differences in the results between an earlier paper by Gibbons and Hull (GH) [34] and the one here: GH failed to find, within a class of non-compact warped supergravity solutions, a sensible 4D de Sitter solution that leads to a finite 4D Planck mass, whereas the one here has achieved this goal. Moreover, within the GH construction, the viable solutions were found to be singular, which were realised by considering a geometry for which the internal space is asymptotic to a cone over a product of spheres, whereas the solutions presented here are non-singular.

\subsection{Neglecting the warp factor}

In this subsection, we momentarily return to the set of $10 \mathrm{D}$ curvature tensors presented in section 2, i.e. equations (2.3) and (2.4), from which we obtain

$$
\begin{aligned}
{ }^{(10)} R_{\mu}^{\mu} & =e^{-\beta A} \hat{R}_{4}-e^{-\alpha A}\left(4\left(\beta^{2}+\beta \alpha\right)(\nabla A)^{2}+2 \beta \nabla^{2} A\right)=\frac{1}{2} T_{4}-\frac{1}{2} T_{6}, \\
{ }^{(10)} R_{i}^{i} & =e^{-\alpha A}\left(\tilde{R}^{(6)}-\left(5 \alpha^{2}+4 \beta \alpha+\beta^{2}\right)(\nabla A)^{2}-(2 \beta+5 \alpha) \nabla^{2} A\right)=\frac{1}{4} T_{6}-\frac{3}{4} T_{4} .
\end{aligned}
$$

From these equations, along with equation (2.3), we derive

$$
\begin{aligned}
\hat{G}_{\mu \nu} & =\hat{R}_{\mu \nu}-\frac{1}{2} \hat{g}_{\mu \nu} \hat{R}_{4} \\
& =T_{\mu \nu}+\frac{\hat{g}_{\mu \nu}}{2}\left(\frac{1}{4} T_{6}-\frac{3}{4} T_{4}\right) e^{\beta A}-\hat{g}_{\mu \nu}\left(\left(\beta^{2}+\beta \alpha\right)(\nabla A)^{2}+\frac{\beta}{2} \nabla^{2} A\right) e^{(\beta-\alpha) A} \\
& =T_{\mu \nu}+\frac{\hat{g}_{\mu \nu}}{2}\left(\tilde{R}^{(6)}-\left(5 \alpha^{2}+6 \beta \alpha+3 \beta^{2}\right)(\nabla A)^{2}-(3 \beta+5 \alpha) \nabla^{2} A\right) e^{(\beta-\alpha) A} .(2 .
\end{aligned}
$$

\footnotetext{
${ }^{6}$ In [32], Greene et al. argued that models of compactifications with both a large volume and a large mass gap may produce phenomenologically acceptable inflationary models with less number of fine tunings, and this might actually be the case also in the present construction once one supplements the gravitational action with a scalar potential.
} 
In the limit $A(y) \rightarrow 0$, or that $A(y) \rightarrow A_{0}$, in that later case the constant term $e^{(\beta-\alpha) A_{0}}$ can be absorbed into $\tilde{R}^{(6)}$ ), the above expression reduces to (see also [25])

$$
\hat{R}_{\mu \nu}-\frac{1}{2} \hat{g}_{\mu \nu} \hat{R}=T_{\mu \nu}+\frac{1}{2} \hat{g}_{\mu \nu} \tilde{R}^{(6)} .
$$

Evidently, in the limit $A(y) \rightarrow 0$, one has $R_{4}=\hat{R}_{4}, R_{6}=\tilde{R}_{6}$ and hence

$$
\hat{R}_{4}=\frac{1}{2}\left(T_{4}-T_{6}\right), \quad \tilde{R}_{6}=\frac{1}{4}\left(T_{6}-3 T_{4}\right),
$$

and

$$
\hat{R}_{4}=-T_{4}-2 \tilde{R}_{6} .
$$

This result shows that in a universe dominated by radiation, for which $a(t) \propto t^{1 / 2}$ and $T_{4}=0$, the magnitude of the $4 \mathrm{D}$ Ricci scalar is just two times the magnitude of $6 \mathrm{D}$ Ricci scalar, but with opposite sign. This implies that, in the absence of warping, the extra dimensional manifold remains equally large as the physical $4 \mathrm{D}$ universe, leading to a phenomenologically unacceptable scenario.

A pertinent question to ask is: Can we get de Sitter solutions just by adding background fluxes, but dropping the warp factor? The answer seems to be negative despite the occurrence of one or more extra terms on the right-hand side of equation (2.43) or equation (2.45).

\section{Effects of flux}

In this section, we consider a $D$-dimensional Einstein action coupled to p-form gauge field strengths or matter fields:

$$
S=\int \sqrt{-g}\left(R^{(D)}-\frac{1}{2} \sum_{p}\left|F_{(p)}\right|^{2}\right) .
$$

Here, as in $[13,25]$, we follow a non-standard normalisation of $\left|F_{(p)}\right|^{2}$, which allows one to treat the $p=0$ and $p>0$ cases uniformly.

\subsection{Magnetic flux}

The energy-momentum tensor due to p-form field strengths is given by

$$
T_{A B}=p F_{A Q_{1} Q_{2} \cdots Q_{p-1}} F_{B} Q_{1} Q_{2} \cdots Q_{p-1}-\frac{1}{2} g_{A B} F_{p}^{2} .
$$

With p-form magnetic flux (M), only the $(i j)$ components of $F_{p}$ are non-vanishing. Since $F_{p}$ depends only on internal space coordinates, we obtain

$$
T_{\mu \nu}^{(M)}=-\frac{1}{2} g_{\mu \nu} F_{p}^{2}, \quad T_{i j}=p F_{i m_{1} m_{2} \cdots m_{p-1}} F_{j}{ }^{m_{1} m_{2} \cdots m_{p-1}}-\frac{1}{2} g_{i j} F_{p}^{2} .
$$

This implies that $T_{4}^{(M)}=-2 F_{p}^{2}, T_{6}^{(M)}=(p-3) F_{p}^{2}$ and hence

$$
R_{6}=\frac{1}{4} T_{6}-\frac{3}{4} T_{4}=\frac{p+3}{4} F_{p}^{2}
$$

That is, a magnetic flux contributes to $R_{6}$ positively. 


\subsection{Electric flux}

Here we consider the effect of an electric flux. In the case of p-form electric flux (E) with $p \geq 4$, an appropriate ansatz is

$$
F_{\mu \lambda \rho \sigma q_{1} q_{2} \cdots q_{p-4}}=i \epsilon_{\mu \lambda \rho \sigma} f_{q_{1} q_{2} \cdots q_{p-4}} .
$$

The four components (or legs) of $F_{p}$ are in usual $4 \mathrm{D}$ spacetime and the rest are in internal spaces. This yields

$$
\begin{array}{r}
T_{\mu \nu}^{(E)}=-\frac{1}{2} g_{\mu \nu} p(p-1)(p-2)(p-3) f_{q_{1} \cdots q_{p-4}} f^{q_{1} \cdots q_{p-4}} \equiv-\frac{1}{2} g_{\mu \nu} \tilde{f}_{p}^{2}, \\
T_{i j}^{(E)}=-p(p-1)(p-2)(p-3)(p-4) f_{i q_{1} q_{2} \cdots q_{p-5}} f_{j}{ }^{q_{1} q_{2} \cdots q_{p-5}}+\frac{1}{2} g_{i j} \tilde{f}_{p}^{2} .
\end{array}
$$

From these we obtain

$$
T_{4}^{(E)}=-2 \tilde{f}_{p}^{2}, \quad T_{6}^{(E)}=(7-p) \tilde{f}_{p}^{2}
$$

and hence

$$
R_{6}=\frac{1}{4} T_{6}-\frac{3}{4} T_{4}=\frac{13-p}{4} \tilde{f}_{p}^{2} \equiv \frac{\tilde{p}+3}{4} \tilde{f}_{10-\tilde{p}}^{2}
$$

In the last line above we replaced $p$ by $(10-\tilde{p})$. Comparing this with $(3.4)$ reveals that an electric contribution is dual to a magnetic flux. As is evident, the replacement

$$
p \rightarrow(10-p), \quad F_{p}^{2} \rightarrow \tilde{f}_{p}^{2}
$$

is nothing but a symmetry between magnetic and electric $p$-form field strengths.

In addition to the background p-form field strengths, one could also introduce some localized objects or brane sources (with $p \geq 3$ ), in some subspaces of the internal manifold $\mathcal{M}$ :

$$
S_{\mathrm{loc}}=-\tau_{p} \int_{M_{4} \times \mathcal{M}} d^{p+1} \xi \sqrt{-\tilde{g}}+\mu_{p} \int_{M_{4} \times \mathcal{M}} C_{p+1},
$$

where $\tau_{p}$ is the p-brane tension, $C_{p+1}$ represents Chern-Simon terms and the pull-back metric $\tilde{g}$ is defined through

$$
\tilde{g}_{\mu \nu} \equiv \frac{\partial X^{A}}{\partial \xi_{\mu}} \frac{\partial X^{B}}{\partial \xi_{\nu}} g_{A B}
$$

In this case, $R_{4}$ and $R_{6}$ each term receives an extra contribution:

$$
R_{4}^{\mathrm{loc}}=\frac{p-7}{4} \tau_{p} \delta(\mathcal{M}), \quad R_{6}^{\mathrm{loc}}=\frac{15-p}{4} \tau_{p} \delta(\mathcal{M}) .
$$

The existence of delta function $\delta(\mathcal{M})$ implies that the energy momentum tensor for the local sources is localized (or confined) within the internal manifold $\mathcal{M}$ or its subspace.

\subsection{Total effects}

Combining the above results, in spacetime dimensions $D=10$, one finds $[13,25]$

$$
\begin{aligned}
{ }^{(10)} R_{\mu}^{\mu} & \equiv R_{4}=-\frac{p-1}{2} F_{p}^{2}+\frac{p-9}{2} \tilde{f}_{p}^{2}+\frac{p-7}{4} \tau_{p} \delta(\mathcal{M}), \\
{ }^{(10)} R_{i}^{i} & \equiv R_{6}=\frac{p+3}{4} F_{p}^{2}+\frac{13-p}{4} \tilde{f}_{p}^{2}+\frac{15-p}{4} \tau_{p} \delta(\mathcal{M}) .
\end{aligned}
$$


Here $0<p \leq 6,4 \leq p \leq 9$ and $p \geq 3$, respectively, in the first, second and third terms on the right-hand side. Since $3 \leq p \leq 9$ (in $D=10$, a D9-brane fills up whole of the spacetime), $R^{(6)}$ is always positive, except when $\tau_{p}$ takes a large but negative value. However, as we explicitly show below, the condition $R^{(6)}>0$ does not necessarily require that $\tilde{R}_{6}>0$ except when the warp factor becomes a constant.

By combining equations (3.11a) and (3.11b), we obtain

$$
R_{10}=R_{4}+R_{6}=\frac{5-p}{4}\left(F_{p}^{2}-\tilde{f}_{p}^{2}\right)+2 \tau_{p} \delta(\mathcal{M}) .
$$

This shows that a self-dual 5 -form field contributes to $R_{4}$ and $R^{(6)}$ with opposite signs and its overall contribution to $10 \mathrm{D}$ Ricci curvature is zero.

\section{Incorporating the warp factor}

Let us first consider the effects of a q-form magnetic field. In this case, using the co-ordinate transformation, $\sinh y d y \rightarrow d z$, it would be convenient to write the 10-dimensional metric ansatz in the following form

$$
d s_{4+q}^{2}=e^{\beta A(z)} \hat{g}_{\mu \nu} d x^{\mu} d x^{\nu}+e^{\alpha A(z)}\left(d z^{2}+s(z)^{2} d X_{q}^{2}\right),
$$

where $s(z)=z+$ const $=\cosh y>0$ is a positive definite function of $z$. Although in the string/M theory case one would set $q=5$ ( or $q=6$ ), let us keep it arbitrary, for generality.

From Maxwell's equation $\partial_{A}\left(\sqrt{-g} F^{A Q_{1} \cdots Q_{q}}\right)=0$, we obtain

$$
F^{A Q_{1} \cdots Q_{q}} \propto(-g)^{-1 / 2}=b s^{-q} \exp \left[\left(-\frac{(q+1) \alpha}{2}-2 \beta\right) A\right]
$$

where $b$ is a constant. The q-form (magnetic) field strength may be written as [35]

$$
F_{q}=b \exp \left[\left(\frac{(q+1) \alpha}{2}-2 \beta\right) A(z)\right] s^{q}(z) d z \wedge d \Omega_{q}
$$

and hence

$$
F_{q}^{2}=b^{2} e^{-4 \beta A} .
$$

Through equation (3.11b), we learned that the $q$-form fluxes (with $q \leq 9$ ) contribute negatively to $R^{(4)}$ and positively to $R^{(6)}$. From this one may erroneously conclude that in the presence fluxes, there may exist a $4 \mathrm{D}$ de Sitter solution only when the $6 \mathrm{D}$ curvature $\tilde{R}_{6}$ is positive. However, as we show below, the integrated $6 \mathrm{D}$ curvature $R_{6}$ can be positive even when the $6 \mathrm{D}$ space itself is negatively curved. As a result, the positivity of flux contributions does not necessarily kill solutions having a Ricci flat or negatively curved 6D space.

\subsection{A specific example}

In this subsection we momentarily drop the contribution of fluxes.

The 10D metric ansatz may be written as

$$
d s_{10}^{2}=e^{\beta A} \hat{g}_{\mu \nu} d x^{\mu} d x^{\nu}+e^{\alpha A}\left(e^{2 B} d y^{2}+e^{2 C} d X_{5}^{2}\right)
$$


where $A, B, C$ are some functions of $y$ and

$$
\begin{aligned}
d X_{5}^{2} & =\frac{1}{9}\left(d \psi+\sum_{i=1}^{2} \cos \theta_{i} d \phi_{i}\right)^{2}+\frac{1}{6} \sum_{i=1}^{2}\left(d \theta_{i}^{2}+\sin \theta_{i}^{2} d \phi_{i}^{2}\right), \\
& \equiv e_{\psi}^{2}+\left(e_{\theta_{1}}^{2}+e_{\phi_{1}}^{2}+e_{\theta_{2}}^{2}+e_{\phi_{2}}^{2}\right) .
\end{aligned}
$$

Here $\left(\theta_{1}, \phi_{1}\right),\left(\theta_{2}, \phi_{2}\right)$ are coordinates on each $S^{2}, \psi$ is the coordinate of a $U(1)$ fiber, and

$$
e_{\psi}=\frac{1}{3}\left(d \psi+\sum_{i=1}^{2} \cos \theta_{i} d \phi_{i}\right), \quad e_{\theta_{i}}=\frac{d \theta_{i}}{\sqrt{6}}, \quad e_{\phi_{i}}=\frac{\sin \theta_{i} d \phi_{i}}{\sqrt{6}} .
$$

Suppose we have $\alpha=0$. We then find that the 6D metric becomes Ricci flat when

$$
B(y)=C(y)+\ln \frac{d C(y)}{d y} .
$$

The two simple solutions that satisfy the above equation are given by

(i) $B(y)=B_{0}, C(y)=B_{0}+\ln (y+c), \quad$ (ii) $B(y)=\ln \sinh y, C(y)=\ln \cosh y$.

For simplicity, we impose the gauge condition $\beta A \equiv-(B+5 C)$. This choice brings the $10 \mathrm{D}$ metric in an Einstein-conformal frame. A straightforward calculations yields

$$
\begin{array}{r}
R^{(4)} \equiv{ }^{(10)} R_{\mu}^{\mu}=e^{B+5 C} \hat{R}_{4}-4 e^{-2 B}\left[\frac{3}{2}{B^{\prime}}^{2}+\frac{25}{2} C^{\prime 2}+10 B^{\prime} C^{\prime}-\frac{1}{2}(B+5 C)^{\prime \prime}\right], \\
R^{(6)} \equiv{ }^{(10)} R_{i}^{i}=e^{-2 C}\left[20+e^{2(C-B)}\left(2 B^{\prime \prime}-5 C^{\prime 2}-3 B^{\prime 2}\right)\right],
\end{array}
$$

where $^{\prime} \equiv \partial / \partial y$. As an illustrative case, let us take ${ }^{7}$

$$
B(y) \equiv \ln \sinh y, \quad C(y) \equiv \ln \cosh y+\ln c .
$$

The 6D Ricci curvature and (Riemann) $)^{2}$ terms are now given by

$$
\tilde{R}_{6}=\frac{20\left(1-c^{2}\right)}{c^{2} \cosh ^{2} y}, \quad R_{i j k l} R^{i j k l}=\frac{8\left(5 c^{4}-10 c^{2}+17\right)}{c^{4} \cosh ^{4} y} .
$$

Furthermore,

$$
R^{(6)}=\frac{20}{\cosh ^{2} y} \frac{1}{c^{2}}+\frac{2}{\sinh ^{2} y}-\frac{5}{\cosh ^{2} y}-\frac{5 \cosh ^{2} y}{\sinh ^{4} y}
$$

and

$$
R_{y \rightarrow \infty}^{(4)} \rightarrow \frac{\hat{R}_{4}}{64} e^{6 y}-\left(416-\frac{20}{c^{2}}\right) e^{-2 y}, \quad R_{y \rightarrow \infty}^{(6)} \rightarrow\left(\frac{80}{c^{2}}-32\right) e^{-2 y} .
$$

In the range $0<c^{2}<5 / 2$, which includes all three possibilities: $\tilde{R}_{6}>0\left(0<c^{2}<1\right)$, $\tilde{R}_{6}=0\left(c^{2}=1\right)$ and $\tilde{R}_{6}<0\left(1<c^{2}<5 / 2\right)$, the integrated $6 \mathrm{D}$ curvature $R^{(6)}$ is positive. In this particular example, $R^{(6)}$ takes a negative value as $y \rightarrow 0$. Based on the result $(3.11 \mathrm{~b})$, one sees the necessity of certain localized sources, such as, $O 6$ planes or negative tension objects. Nevertheless, in the limit $y \rightarrow 0$, since $\sqrt{g_{6}} \rightarrow 0$, the supergravity approximations also break down. For consistency, one then needs to supplement the Einstein-Hilbert term in the action by higher curvature and/or higher derivative terms with some coefficients or coupling constants, whose positivity is not guaranteed. That is, a gravitational Lagrangian with higher curvature terms (or stringy corrections) will not in general lead to a strong restriction on the sign of $R^{(6)}$.

\footnotetext{
${ }^{7}$ This choice does not particularly help us to solve the $10 \mathrm{D}$ supergravity equations analytically, but it is nonetheless useful for studying some qualitative features of $4 \mathrm{D}$ de Sitter solutions by allowing the $6 \mathrm{D}$ manifold to take a negative $\left(c^{2}>1\right)$, zero $\left(c^{2}=1\right)$ or positive $\left(c^{2}<1\right)$ Ricci curvature.
} 


\subsection{A more general example}

The 4D de Sitter solutions can be available in a more general case as well. Consider the following $10 \mathrm{D}$ action:

$$
S_{10}=\frac{1}{2 \kappa_{10}^{2}} \int \sqrt{-g_{10}}\left(R_{10}-\frac{1}{12} F_{3}^{2}-\frac{1}{12} H_{3}^{2}-\frac{1}{4 \cdot 5 !} F_{5}^{2}\right)+S_{\mathrm{CS}},
$$

where $H_{3}$ and $F_{3}$ are 3 -form fields, $F_{5}$ is a self-dual 5 -form field and $S_{\mathrm{CS}}$ denotes the type II Chern-Simons terms. As compared to the gravitational action considered in [30], we have set the $10 \mathrm{D}$ dilaton $\Phi$ and the Ramond-Ramond scalar $\mathcal{C}$ to be zero.

The majority of $10 \mathrm{D}$ constructions made by using a conifold type compactification of type IIB supergravity utilise a non-compact extra dimension. However, most works along this line failed to reproduce a sensible 4D cosmology which requires a finite 4D Planck mass or a finite $6 \mathrm{D}$ warped volume. Here we report on a class of $4 \mathrm{D}$ de Sitter solutions which are not only completely regular everywhere but also lead to an explicit realisation of four-dimensional cosmology with a finite Newton's constant.

We begin with a sufficiently general 10D metric ansatz of the form

$$
d s_{10}^{2}=e^{\beta A} \hat{g}_{\mu \nu} d x^{\mu} d x^{\nu}+e^{-\beta A}\left(e^{10 B} d y^{2}+r^{2} e^{2 B} d X_{5}^{2}\right)
$$

where $r$ is an arbitrary constant, which measures the radius of $X_{5}$. A similar choice was made in the literature before, see, e.g. $[30,37]$. Next, using the freedom to redefine $A(y)$, we set may $\beta=2$. The metric ansatz then takes the following form

$$
d s_{10}^{2}=e^{2 A} \hat{g}_{\mu \nu} d x^{\mu} d x^{\nu}+e^{-2 A}\left(e^{10 B(y)} d y^{2}+r^{2} e^{2 B(y)} d X_{5}^{2}\right),
$$

As compared to some similar models discussed in the literature, here we have made two important generalizations. Firstly, we have allowed the $4 \mathrm{D}$ spacetime to be a maximally symmetric, implying that it is either flat $\hat{R}_{4}=0$, de Sitter $\hat{R}_{4}>0$ or anti de Sitter $\hat{R}_{4}<0$. Secondly, we have allowed an extra (length) parameter $r$ into the 10D metric (equation (4.15)), so that the internal 6D space is also not necessarily flat, which is the case only if $r=1$.

The appropriate ansätze for the p-form fields are now given by

$$
\begin{aligned}
F_{5} & =\mathcal{F}+* \mathcal{F}, \\
\mathcal{F} & =K(y)\left(e_{\psi} \wedge e_{\theta_{1}} \wedge e_{\phi_{1}} \wedge e_{\theta_{2}} \wedge e_{\phi_{2}}\right), \\
* \mathcal{F} & =\frac{e^{8 A}}{r^{5}} K(y) \sqrt{-\operatorname{det} \hat{g}_{\mu \nu}} d y \wedge d x^{0} \wedge d x^{1} \wedge d x^{2} \wedge d x^{3},
\end{aligned}
$$

along with the following constrain equations or Bianchi identities:

$$
\begin{aligned}
& H_{3}=\partial B_{2}=3 \partial_{[P} B_{Q R]}, \quad B_{2}=f(y)\left(e_{\theta_{1}} \wedge e_{\phi_{1}}-e_{\theta_{2}} \wedge e_{\phi_{2}}\right), \\
& F_{3}=\partial C_{2} \equiv c_{1} e_{\psi} \wedge\left(e_{\theta_{1}} \wedge e_{\phi_{1}}-e_{\theta_{2}} \wedge e_{\phi_{2}}\right), \quad d_{*} F_{5}=d F_{5}=H_{3} \wedge F_{3},
\end{aligned}
$$

where $c_{1}$ is an arbitrary constant and $\mathrm{f}(\mathrm{y})$ a general function of $\mathrm{y}$. It is not difficult to check that, with $r^{2}=1$ in equation (4.15), the internal 6D space becomes Ricci flat when

$$
-B^{\prime \prime}+2 B^{\prime 2}+2 e^{8 B}=0 .
$$


This equation is solved by

$$
B(y)=-\frac{1}{8} \ln (4 y+b)^{2} .
$$

Here, since $y$ ranges from 0 to $\infty$, it is mandatory to assume that $b>0$, so that the $6 \mathrm{D}$ space is regular everywhere. Furthermore,

$$
\tilde{R}_{6}=\frac{20\left(1-r^{2}\right) e^{-2 B}}{r^{2}}, \quad \tilde{R}_{i j k l} \tilde{R}^{i j k l}=\left(\frac{136}{r^{4}}-\frac{80 b}{r^{2}}+40\right) e^{-4 B},
$$

which both are regular at $y=0$. Since $0 \leq y<\infty$, we have

$$
0<r^{2}<\infty
$$

which includes all three possibilities that $0<r^{2}<1$ (positively curved $6 \mathrm{D}$ space), $r^{2}=1$ (Ricci flat $6 \mathrm{D}$ space) and $1<r^{2}<\infty$ (negatively curved 6D space).

By evaluating flux contributions to the 10D supergravity action, we explicitly find

$$
\begin{gathered}
S_{\text {eff }}=\frac{M_{10}^{2}}{2} \operatorname{Vol}\left(X_{5}\right) r^{5} \int d y \int \sqrt{-\hat{g}_{4}} d^{4} x\left[\frac{1}{(4 y+b)^{5 / 2}}\left(e^{-4 A} \hat{R}_{4}+\tilde{R}_{6}\right)+2 A^{\prime \prime}-8 A^{\prime 2}\right. \\
\left.-\frac{c_{1}^{2}}{(4 y+b) r^{6}} e^{4 A}-\frac{(4 y+b)}{r^{4}} e^{4 A}\left(\partial_{y} f\right)^{2}-\frac{\left(c_{0}+2 c_{1} f(y)\right)^{2}}{2 r^{10}} e^{8 A}\right] .
\end{gathered}
$$

Indeed, the second order (nonlinear) equations of motion derived from an effective 4D lagrangian, but ignoring the second derivative terms, such as $A^{\prime \prime}(y)$, or $z^{\prime \prime}$ in the notation of [37], do not necessarily solve the full system of 10D supergravity equations. We shall therefore consider the original system of 10D Einstein equations, which reduce to ${ }^{8}$

$$
\begin{gathered}
e^{-4 A} \hat{R}_{4}-4(4 y+b)^{5 / 2} A^{\prime \prime}=-(4 y+b)^{3 / 2} e^{4 A}\left(\frac{c_{1}^{2}}{r^{6}}+\frac{(4 y+b)^{2}}{r^{4}}\left(\partial_{y} f\right)^{2}+\frac{\mathcal{F}^{2}(4 y+b)}{2 r^{10}} e^{4 A}\right) \\
20 \sqrt{(4 y+b)}\left(\frac{1-r^{2}}{r^{2}}\right)+(4 y+b)^{5 / 2}\left(6 A^{\prime \prime}-8 A^{\prime 2}\right) \\
=(4 y+b)^{3 / 2} e^{4 A}\left(\frac{3}{2} \frac{c_{1}^{2}}{r^{6}}+\frac{3(4 y+b)^{2}}{2 r^{4}}\left(\partial_{y} f\right)^{2}+\frac{\mathcal{F}^{2}(4 y+b)}{2 r^{10}} e^{4 A}\right)
\end{gathered}
$$

where $\mathcal{F} \equiv c_{0}+2 c_{1} f(y)$. The problem simplifies a lot in the case $\hat{R}_{4}=0$ and $r^{2}=1$. This case has been extensively studied in the literature, see, for example, [15,37].

For $0<r^{2}<1$ and $r^{2}=1$, which correspond, respectively, to the positively curved and Ricci flat $6 \mathrm{D}$ spaces, there is no large $r$ limit solution. As a result the flux contributions may not be negligible even when one takes a large $y$ limit. However, in the case of a negatively curved 6D space, the 5-form flux contributions can be small in the large $r$ limit. Moreover, as with standard compact hyperbolic manifolds, the 6D curvature and Kaluza-Klein mass gap may be fixed by two physical parameters ${ }^{9}$. The freedom to allow $r^{2}$ in the range $1<r^{2}<\infty$, or a negatively curved 6D space, is an important difference with respect to the case of the sphere or Ricci flat $6 \mathrm{D}$ space, where the internal volume and Kaluza-Klein mass gap are generally fixed in terms one parameter, the radius of $\mathcal{M}=X_{6}$.

\footnotetext{
${ }^{8}$ In our case, it is sufficient to consider only the trace parts of $10 \mathrm{D}$ Einstein equations.

${ }^{9}$ See, for example, ref. [36] for a discussion related to phenomenological advantages of using hyperbolic extra dimensions.
} 


\subsection{Vacuum case}

In the absence of fluxes, i.e. in the absence of terms on the right-hand side of equations (4.22) and (4.23), the system of equations are explicitly solved when ${ }^{10}$

$$
r^{2}=2, \quad \hat{R}_{4}=8 e^{4 a_{1}}, \quad A(y)=-\frac{1}{8} \ln (4 y+b)+a_{1} .
$$

The explicit 10D metric solution is given by

$$
d s_{10}^{2}=e^{2 A(y)}\left[\hat{g}_{\mu \nu} d x^{\mu} d x^{\nu}+2 e^{-4 a_{1}}\left(\frac{d y^{2}}{2(4 y+b)^{2}}+d X_{5}^{2}\right)\right] .
$$

In this case, the extra dimensions are hyperbolic (or negatively curved). Here, the y coordinate varies from 0 to $\infty$. Of course, the $6 \mathrm{D}$ volume (without the effect of warping), which is given by $V_{6} \sim \int \sqrt{g_{6}} d^{6} y \sim \operatorname{Vol}\left(X_{5}\right) \int(4 y+b)^{-1} \propto \ln (4 y+b)$ grows logarithmically with $y$. However, when we take into account the effect of warp factor (or warping of extra dimensions), then the $6 \mathrm{D}$ warped volume, which is given by

$$
V_{6}^{\text {warped }} \sim \operatorname{Vol}\left(X_{5}\right) \int_{0}^{\infty} \frac{d y}{(4 y+b)^{2}} \propto \operatorname{Vol}\left(X_{5}\right)\left[\frac{-1}{4 y+b}\right]_{0}^{\infty}=\frac{\operatorname{Vol}\left(X_{5}\right)}{b}
$$

is finite. The solution therefore leads to a finite $4 \mathrm{D}$ effective Planck mass.

\subsection{Effects of fluxes}

In the presence of both 3 -form and 5-form fields, we may analyse the system of $10 \mathrm{D}$ supergravity equations by imposing the condition ${ }^{11}$

$$
g_{s}^{2} F_{3}^{2}=H_{3}^{2},
$$

where $g_{s}$ is string coupling constant. We then find

$$
f(y)=\sqrt{\frac{g_{s}^{2} c_{1}^{2}}{16 r^{2}}} \ln (4 y+b)+\frac{f_{0}}{|r|},
$$

where $f_{0}$ is a constant. We now have

$$
\begin{aligned}
& R^{(4)}=e^{-2 A} \hat{R}_{4}-4(4 y+b)^{5 / 2} A^{\prime \prime} e^{2 A} \\
&=-f_{1}(4 y+b)^{3 / 2} e^{6 A}-\left(f_{2}+f_{3} \ln (4 y+b)\right)^{2}(4 y+b)^{5 / 2} e^{10 A},(4) \\
& R^{(6)}=20 \sqrt{(4 y+b)}\left(\frac{1-r^{2}}{r^{2}}\right) e^{2 A}+(4 y+b)^{5 / 2}\left(6 A^{\prime \prime}-8 A^{\prime 2}\right) e^{2 A} \\
&=\frac{3 f_{1}}{2}(4 y+b)^{3 / 2} e^{6 A}+\left(f_{2}+f_{3} \ln (4 y+b)\right)^{2}(4 y+b)^{5 / 2} e^{10 A} .(4 .
\end{aligned}
$$

\footnotetext{
${ }^{10}$ With $r^{2}=1$ (and hence $\tilde{R}_{6}=0$ ), which are the original choice made by Kachru el al. [15], there exists only a trivial solution with $A(y)=$ const and $\hat{R}_{4}=0$. The non-existence of a $4 \mathrm{D}$ de Sitter within the framework of [15] and in many follow up works was just due to the use of some oversimplified 10D metric Ansätze.

${ }^{11}$ This relationship is typically obtained in type II supergravity by taking a variation of $10 \mathrm{D}$ dilaton $\Phi$ and then by taking the limit where $\Phi \rightarrow$ const.
} 
The coefficients like $f_{1}, f_{2}$ and $f_{3}$ are not fully determined, except that

$$
f_{1}=\frac{c_{1}^{2}}{r^{6}}\left(1+g_{s}^{2}\right)>0, \quad f_{3}=\frac{c_{1}^{2} g_{s}}{2 r^{6}}>0,
$$

while the coefficient $f_{2} \equiv\left(c_{0} r+2 c_{1} f_{0}\right) / r^{6}$ can take any values. Our aim here is not to solve the equations (4.28)-(4.29) exactly, which is anyway not possible ${ }^{12}$ but to study the possible effects of flux terms on the $4 \mathrm{D}$ scalar curvature $\hat{R}_{4}$ and also on $R^{(6)}$.

The 3 -form flux scales as the energy source for the curvature when

$$
e^{4 A} \equiv \frac{e^{4 a_{1}}}{4 y+b}
$$

This leads to

$$
R^{(6)}=\frac{4\left(5-r^{2}\right)}{r^{2}} e^{4 a_{1}}=\frac{3 f_{1}}{2} e^{6 a_{1}}+\left(f_{1}+f_{3} \ln (4 y+b)\right)^{2} e^{10 a_{1}} .
$$

We are now required to take $r^{2}<5$. This includes the possibility of having a negatively curved $6 \mathrm{D}$ space, i.e. $r^{2}>1$. From the expression of $R^{(4)}$ we can see that $\hat{R}_{4}>0$ provided that

$$
16-f_{1} e^{4 a_{1}}-\left(f_{2}+f_{3} \ln (4 y+b)\right)^{2} e^{8 a_{1}}>0 .
$$

This can be satisfied by taking small $f_{i} \mathrm{~s}(i=1,2,3)$ or that $a_{1} \ll 0^{13}$.

From equations (4.28)-(4.29), we can see that a sufficiently long period of $4 \mathrm{D}$ de Sitter expansion becomes possible when the warp factor varies as

$$
e^{4 A} \equiv \frac{e^{4 a_{1}}}{(4 y+b)^{\gamma}} \quad(\gamma>1)
$$

or more rapidly. In such a case the effect of fluxes fall off more rapidly as compared to the energy sources for the curvature. For illustration, take $\gamma=3 / 2$, so that

$$
R^{(6)}=\frac{2\left(10-r^{2}\right)}{r^{2}(4 y+b)^{1 / 4}} e^{2 a_{1}} .
$$

The positivity of $R^{(6)}$ now requires that $r^{2}<10$. Furthermore

$$
\hat{R}_{4} \rightarrow \frac{96 e^{4 a_{1}}}{(4 y+b)}-f_{1} \frac{e^{8 a_{1}}}{(4 y+b)^{3 / 2}}-\frac{\left(f_{2}+f_{3} \ln (4 y+b)\right)^{2}}{(4 y+b)^{2}} e^{12 a_{1}} .
$$

Qualitatively, we can have three branches of solutions:

$$
\begin{aligned}
0<y<y_{c}: & \hat{R}_{4}>0, \\
y_{c}<y<y_{2}: & \hat{R}_{4}<0, \\
y \rightarrow \infty: & \hat{R}_{4} \sim \frac{e^{4 a_{1}}}{y}\left(24-f_{1} \frac{e^{4 a_{1}}}{8 y^{1 / 2}}+\cdots\right)>0 .
\end{aligned}
$$

\footnotetext{
${ }^{12}$ Indeed, the choice as $\beta=-\alpha$ and $C=5 B+$ const does not help us to analytically solve the 10D supergravity equations except when the flux contributions become negligible or in the limit $\hat{R}_{4} \rightarrow 0$, in the latter case one may supplement the full system of second order (nonlinear) equations with BPS saturated first-order equations as in Klebanov-Strassler work [5] (see also [15,37]), but it nonetheless assists us to simplify the system of field equations.

${ }^{13}$ In this case, one needs to have a relatively large volume for the extra dimension, or a reasonably small value of $c_{1}^{2}$, since otherwise $\hat{R}_{4}$ will change its sign from positive to negative as $y \rightarrow \infty$.
} 
Here, $y_{c}$ is some critical value of $y$, for which the energy sources of the curvature and 3 -form gauges fields become comparable and hence $\hat{R}_{4} \sim 0$. That is, in the presence of fluxes, there could arise an intermediate branch where $\hat{R}_{4}<0$ is possible, thereby generating an epoch of decelerating expansion between two de Sitter expansions ${ }^{14}$. It would be very useful to find an explicit solution of 10D supergravity equations realizing such a behaviour.

\section{A model with compact extra dimensions}

In this section, which may be read independently of the above sections, we will briefly discuss an alternative scenario where the fifth dimension is compact (similar to that in the RS 2-brane model). For simplicity, we will drop all source terms (or fluxes).

It is not difficult to check that the following 11-dimensional metric ansatz

$$
d s_{11}^{2}=e^{K(y)}\left(-d t^{2}+e^{2 m_{4} t} d \mathbf{x}_{3}^{2}+\lambda^{2} \tanh ^{2} y d y^{2}+\rho^{2} d \Omega_{6}^{2}\right),
$$

where $d \Omega_{6}^{2}$ denotes the metric of a standard six-sphere ${ }^{15}$, explicitly solves all of the $11 \mathrm{D}$ Einstein field equations when

$$
K(y)= \pm \sqrt{\frac{20 \lambda^{2}}{9 \rho^{2}}} \ln \cosh y+K_{0}, \quad m_{4}=\sqrt{\frac{5}{3 \rho^{2}}} .
$$

Here we take the negative sign ${ }^{16}$. As the $y$ coordinate is assumed to be a closed cycle, we can write the total action as

$$
S=\frac{M_{11}^{2}}{2} \int d^{11} x \sqrt{-g_{11}} R_{11}+\int_{M^{4} \times \mathcal{M}} \sqrt{-g_{b 1}}\left(-\tau_{b 1}\right)+\int_{M^{4} \times \mathcal{M}} \sqrt{-g_{b 2}}\left(-\tau_{b 2}\right),
$$

where $\tau_{b 1}, \tau_{b 2}$ denote the brane tensions corresponding to the two p-branes $b 1$ and $b 2$, which may be placed at orbifold fixed points $y=0$ and $y=\pi$. Here, we are assuming the existence of something like a D9-brane, i.e. $p=9$, so each brane has 10 dimensions. The 6 spatial directions of p-brane extend in the internal manifold $X_{6}$, while the 4 other directions (including one time-like) extend along the $x^{\mu}$ directions. The Horava-Witten 'world brane' [38] is such a brane.

The solution valid for $-\pi \leq y \leq \pi$ implies that

$$
K^{\prime \prime}+\frac{2 \sqrt{5} \lambda}{3 \rho \cosh ^{2} y}+\frac{2 \sqrt{5} \lambda}{3 \rho} \tanh y(2 \delta(y)-2 \delta(y-\pi))=0 .
$$

From the $\mu \nu$ components of the 11D Einstein equations, we obtain

$$
\begin{aligned}
& \frac{9 e^{-K / 2}}{\lambda \tanh y}\left(K^{\prime \prime}-\frac{2 K^{\prime}}{\sinh (2 y)}+2 K^{\prime 2}-\frac{2 \lambda^{2} \tanh ^{2} y}{3}\left(m_{4}^{2}+\frac{15}{3 \rho^{2}}\right)\right)+\frac{4 \tau_{b 1}}{M_{11}^{2}} \delta(y)+\frac{4 \tau_{b 2}}{M_{11}^{2}} \delta(y-\pi)=0 \\
& \Rightarrow \frac{9 e^{-K / 2}}{\lambda \tanh y}\left(K^{\prime \prime}+\frac{2 \sqrt{5} \lambda}{3 \rho \cosh ^{2} y}\right)+\frac{4 \tau_{b 1}}{M_{11}^{2}} \delta(y)+\frac{4 \tau_{b 2}}{M_{11}^{2}} \delta(y-\pi)=0 .
\end{aligned}
$$

\footnotetext{
${ }^{14}$ The second phase of an accelerating expansion is not purely de Sitter since $\hat{R}_{4}$ is no longer a constant.

${ }^{15}$ One could in principle replace $S^{m}$ by Einstein-Sasaki spaces $\left(S^{m-n} \times S^{n-1}\right) \rtimes S^{1}$ or some other compact manifolds, in a way similar to the 10D case where gravity solutions with $X_{5}=S^{5}$ are physically equivalent to that with $X_{5}=T^{1,1}$, at least, in pure Einstein gravity (i.e. without source terms).

${ }^{16}$ Only the negative sign in this solution leads to a finite $4 \mathrm{D}$ effective Planck mass once the orbifold symmetry around $y=[0, \pi]$ is relaxed. As in the $10 \mathrm{D}$ case, we can get a more general solution by replacing $\cosh y$ by $\cosh y+\epsilon \sinh y$ and $\tanh y$ by $(\epsilon+\tanh y) /(1+\epsilon \tanh y)$.
} 
In the second line above we have substituted the solution (5.2). The $i j$ components $(i, j=$ $6, \cdots, 11$ ) of the 11D Einstein tensor $G_{A B}$ also lead to the same result (cf (5.5)).

By comparing the above set of equations, we explicitly get

$$
\tau_{b 1}=\frac{3 \sqrt{5}}{\rho M_{11}^{2}} e^{-K_{0} / 2}, \quad \tau_{b 2}=-\frac{3 \sqrt{5}}{\rho M_{11}^{2}} e^{-K_{0} / 2} \exp \left(-\frac{5 \lambda}{3 \sqrt{2} \rho} \ln \cosh \pi\right) .
$$

With a suitable choice of the ratio $\lambda / \rho$, one could possibly explain the mass hierarchy problem in particle physics, similar to that in the RS 2-brane model [1]. From (5.1), we can see that the warp factor acquires a low value at $y=\pi$ (compared to that at $y=0)$, which is necessary for the hierarchy solution. The compactification scale $\rho$, which is proportional to the size of $X_{6}$, is not necessarily small within our model. We only require that the ratio $\lambda / \rho$ becomes much larger than unity, e.g. $\lambda / \rho \sim \mathcal{O}(10)$. For instance, with $\lambda / \rho \sim 12$, we obtain $\tau_{b_{1}} /\left|\tau_{b_{2}}\right| \sim e^{34.7} \sim 10^{15}$. In the RS 2-brane model, since $p=3$, the brane is extended only along the $x^{\mu}$ directions. Here, as we are considering a p-brane with $p=9$, the brane is extended also along the internal manifold $X_{6}$. That means, we are comparing the 9-brane tensions at the orbifold fixed points $y=0$ and $y=\pi^{17}$.

Next we briefly discuss about phenomenological constraints on the model. Indeed, the compactification scale $\rho$ must respect some experimental bounds which assert for a low $\rho$. To be precise, we shall consider the mass reduction formula (also known as Gauss's law)

$$
M_{\mathrm{Pl}}^{2}=\frac{M_{(11)}^{9} e^{9 K_{0} / 2}}{(2 \pi)^{7}} V_{7}^{\mathrm{w}}, \quad V_{7}^{\mathrm{w}}=\operatorname{Vol}\left(X_{6}\right) \frac{\rho^{7}}{3 \sqrt{5}}\left[1-(\operatorname{sech} \pi)^{3 \sqrt{5} \lambda / \rho}\right],
$$

which is obtained by considering a dimensional reduction from $D=11$ to $D=4$. The 7D warped volume is well approximated by $V_{7}^{\mathrm{w}} \simeq \operatorname{Vol}\left(X_{6}\right) \rho^{7} /(3 \sqrt{5})$ for $\lambda / \rho \gtrsim 1$. There arises a strong constraint on $\rho$, especially, if one wants to tune $\Lambda_{4}$ to the present value of the cosmological constant, $\Lambda_{4} \sim 10^{-120} M_{\mathrm{Pl}}^{2} \sim 10^{-55} \mathrm{~cm}^{-2}$, which is given here by $\Lambda_{4} \sim m_{4}^{2} \sim$ $1 / \rho^{2}$ (cf equation (5.2)). However, when one applies the model to explain a period of de Sitter expansion in the early universe, then the constraint on $\rho$ is mild and it can easily be satisfied. For instance, with $\rho^{-1} \sim 10^{-5} M_{\mathrm{Pl}}$, we obtain $M_{11} \sim 5.5 \times 10^{-5} M_{\mathrm{Pl}} e^{-K_{0} / 2}$. Alternatively, we may demand that $M_{\mathrm{Pl}}>M_{11} \gtrsim 10^{-15} M_{\mathrm{Pl}}$. Then the bound on $\rho$ turns out to be $0.15 e^{K_{0} / 2} M_{\mathrm{Pl}}>\rho^{-1}>0.79 \times 10^{-20} e^{K_{0} / 2} M_{\mathrm{Pl}}$. In view of these bounds, the explicit solutions found in this paper may be used only to explain a de Sitter expansion in the early universe rather than a late epoch cosmic acceleration.

\section{Summary and further remarks}

Finding de Sitter solutions through warped compactifications of type-II supergravities in 10 or 11 dimensions has often been viewed difficult, except in the cases where one supplements the gravitational action with some stringy sources that violate certain positivity conditions. This is more or less the gist of an argument in several versions of no-go theorem for de Sitter compactifications of classical supergravity theories, with physically compact extra dimensions.

\footnotetext{
${ }^{17}$ Alternatively, one can introduce a combination of D3 and M5 branes and also covariant combinations of delta functions and geometric factors necessary to position the branes. In such a case one is usually required to introduce some local sources concentrated (or localized) in the manifold $X_{6}$ in order to solve $11 \mathrm{D}$ Einstein equations at the brane's positions.
} 
In the literature, see, for example, [39,40], there are arguments about the existence of (universal) de Sitter solutions at tree-level supergravity actions in 10 or 11 dimensions, which may involve only compact extra dimensions. The proofs of existence of $4 \mathrm{D}$ de Sitter solutions in these and several other constructions have been based on certain assumptions under which part of the (super) symmetries are broken, giving rise to an effective (scalar field) potential which allows a local minimum, rather than on exact solutions to classical field equations. In this paper we have found a few sufficiently illustrative examples in which four-dimensional de Sitter solutions arise at a purely classical level.

The most interesting achievement of this paper is the discovery of a new class of simple warped vacuum solutions with a $4 \mathrm{D}$ de sitter part and noncompact internal space with still a finite $4 \mathrm{D}$ Planck mass ${ }^{18}$. It is quite plausible that warped compactifications on Ricci nonflat spaces, including manifolds whose mean curvature is negative, lead to the realization of some new cosmological scenarios in string and supergravity theories.

\section{Acknowledgement}

The author is grateful to Kei-ichi Maeda, Shinji Mukohyama, Hideo Kodama, Nobu Ohta and Thomas Van Riet for useful conversations and correspondences. This work was supported by the Marsden fund of the Royal Society of New Zealand.

\section{References}

[1] L. Randall and R. Sundrum, "A large mass hierarchy from a small extra dimension", Phys. Rev. Lett. 83, 3370 (1999) [arXiv:hep-ph/9905221].

[2] L. Randall and R. Sundrum, "An alternative to compactification", Phys. Rev. Lett. 83, 4690 (1999) [arXiv:hep-th/9906064].

[3] J. M. Cline, C. Grojean and G. Servant, "Cosmological expansion in the presence of extra dimensions", Phys. Rev. Lett. 83, 4245 (1999) [arXiv:hep-ph/9906523].

[4] J. M. Maldacena, "The large N limit of superconformal field theories and supergravity", Adv. Theor. Math. Phys. 2, 231 (1998) [arXiv:hep-th/9711200].

[5] I. R. Klebanov and M. J. Strassler, "Supergravity and a confining gauge theory: Duality cascades and chiSB-resolution of naked singularities," JHEP 0008, 052 (2000) [arXiv:hep-th/0007191].

[6] S. Kachru, R. Kallosh, A. Linde and S. P. Trivedi, "De Sitter vacua in string theory", Phys. Rev. D 68, 046005 (2003) [arXiv:hep-th/0301240].

[7] C. P. Burgess, J. M. Cline, H. Stoica and F. Quevedo, JHEP 0409, 033 (2004).

[8] S. Panda, M. Sami and S. Tsujikawa, "Prospects of inflation in delicate D-brane cosmology", Phys. Rev. D 76, 103512 (2007) [arXiv:0707.2848 [hep-th]].

[9] M. Becker, L. Leblond and S. E. Shandera, "Inflation from Wrapped Branes", Phys. Rev. D 76, 123516 (2007) [arXiv:0709.1170 [hep-th]].

[10] D. Cassani and A. K. Kashani-Poor, "Exploiting $\mathrm{N}=2$ in consistent coset reductions of type IIA", Nucl. Phys. B 817, 25 (2009) [arXiv:0901.4251].

[11] D. Lust and D. Tsimpis, "Classes of AdS4 type IIA/IIB compactifications with SU(3)xSU(3) structure", JHEP 0904, 111 (2009) [arXiv:0901.4474].

\footnotetext{
${ }^{18}$ In a recent paper [33], it has been shown that the class of de Sitter solutions found in this paper also lead to a normalizable zero-mass graviton wave-function in four dimensions.
} 
[12] G. W. Gibbons, "Aspects of Supergravity Theories", GIFT Seminar 1984, pp. 123-146, (QCD161:G2:1984), Print-85-0061 (CAMBRIDGE).

[13] B. de Wit, D. J. Smit and H.D. Hari Das, "Residual Suppersymmetry of Compactified $D=10$ Supergravity", Nucl. Phys. B 283, 165 (1987).

[14] J. M. Maldacena and C. Nunez, "Supergravity description of field theories on curved manifolds and a no go theorem," Int. J. Mod. Phys. A 16, 822 (2001).

[15] S. B. Giddings, S. Kachru and J. Polchinski, "Hierarchies from fluxes in string compactifications", Phys. Rev. D 66, 106006 (2002).

[16] C. M. Chen, D. V. Gal'tsov and M. Gutperle, "S-brane solutions in supergravity theories", Phys. Rev. D 66, 024043 (2002) [arXiv:hep-th/0204071].

[17] P. K. Townsend and M. N. R. Wohlfarth, "Accelerating cosmologies from compactification", Phys. Rev. Lett. 91, 061302 (2003) [arXiv:hep-th/0303097].

[18] N. Ohta, "Accelerating cosmologies from S-branes", Phys. Rev. Lett. 91, 061303 (2003) [arXiv:hep-th/0303238];

[19] I. P. Neupane and D. L. Wiltshire, "Accelerating cosmologies from compactification with a twist", Phys. Lett. B 619, 201 (2005) [arXiv:hep-th/0502003]; ibid, "Cosmic acceleration from M theory on twisted spaces", Phys. Rev. D 72, 083509 (2005) [arXiv:hep-th/0504135].

[20] C. M. Chen, P. M. Ho, I. P. Neupane, N. Ohta and J. E. Wang, "Hyperbolic space cosmologies", JHEP 0310, 058 (2003) [arXiv:hep-th/0306291];

I. P. Neupane, "Accelerating cosmologies from exponential potentials", Class. Quant. Grav. 21, 4383 (2004) [arXiv:hep-th/0311071].

[21] K. i. Maeda, N. Ohta, M. Tanabe and R. Wakebe, "Supersymmetric Intersecting Branes in Time-dependent Backgrounds", JHEP 0906, 036 (2009) [arXiv:0903.3298 [hep-th]];

K. i. Maeda, N. Ohta and K. Uzawa, "Dynamics of intersecting brane systems Classification and their applications", JHEP 0906, 051 (2009) [arXiv:0903.5483 [hep-th]].

[22] C. M. Chen, P. M. Ho, I. P. Neupane and J. E. Wang, "A note on acceleration from product space compactification", JHEP 0307, 017 (2003) [arXiv:hep-th/0304177].

[23] R. Emparan and J. Garriga, "A note on accelerating cosmologies from compactifications and S-branes", JHEP 0305, 028 (2003) [arXiv:hep-th/0304124].

[24] I. P. Neupane, "Accelerating universes from compactification on a warped conifold", Phys. Rev. Lett. 98, 061301 (2007) [arXiv:hep-th/0609086].

[25] M. R. Douglas and R. Kallosh, "Compactification on negatively curved manifolds", JHEP 1006, 004 (2010) [arXiv:1001.4008 [hep-th]].

[26] I. P. Neupane, "Simple cosmological de Sitter solutions on $\mathrm{dS}_{4} \times Y_{6}$ spaces", Class. Quant. Grav. 27, 045011 (2010) [arXiv:0901.2568];

I. P. Neupane, "Accelerating universe from warped extra dimensions", Class. Quant. Grav. 26, 195008 (2009) [arXiv:0905.2774].

[27] S. Randjbar-Daemi and M. E. Shaposhnikov, "On some new warped brane world solutions in higher dimensions", Phys. Lett. B 491, 329 (2000) [arXiv:hep-th/0008087];

A. Flachi, J. Garriga, O. Pujolas and T. Tanaka, "Moduli stabilization in higher dimensional brane models", JHEP 0308, 053 (2003) [arXiv:hep-th/0302017];

Y. Aghababaie et al., "Warped brane worlds in six dimensional supergravity", JHEP 0309, 037 (2003) [arXiv:hep-th/0308064];

V. Dzhunushaliev, V. Folomeev and M. Minamitsuji, "Thick de Sitter brane solutions in higher dimensions", Phys. Rev. D 79, 024001 (2009) [arXiv:0809.4076 [gr-qc]].

[28] I. P. Neupane, "Extra dimensions, warped compactifications and cosmic acceleration", Phys. Lett. B 683, 88 (2010) [arXiv:0903.4190]; ibid, "The warping of extra spaces accelerates the expansion of the universe", arXiv:1004.0254 [gr-qc], Received An Honorable Mention in the 2010 Gravity Research Foundation Eassay Competition. 
[29] U. H. Danielsson, S. S. Haque, G. Shiu and T. Van Riet, "Towards Classical de Sitter Solutions in String Theory", JHEP 0909, 114 (2009) [arXiv:0907.2041 [hep-th]].

[30] I. R. Klebanov and A. A. Tseytlin, "Gravity Duals of Supersymmetric SU(N) x SU(N+M) Gauge Theories", Nucl. Phys. B 578, 123 (2000) [arXiv:hep-th/0002159].

[31] Y. M. Cho and I. P. Neupane, "Warped brane-world compactification with Gauss-Bonnet term", Int. J. Mod. Phys. A 18, 2703 (2003) [arXiv:hep-th/0112227].

[32] B. Greene, D. Kabat, J. Levin and D. Thurston, "A bulk inflaton from large volume extra dimensions", arXiv:1001.1423 [hep-th].

[33] I. P. Neupane, "Warped compactification to de Sitter space", Nucl. Phys. B847, 549-566 (2011) [arXiv:1011.5007].

[34] G. W. Gibbons and C. M. Hull, arXiv:hep-th/0111072.

[35] S. Kinoshita and S. Mukohyama, JCAP 0906, 020 (2009) [arXiv:0903.4782 [hep-th]].

[36] D. Orlando and S. C. Park, "Compact hyperbolic extra dimensions: a M-theory solution and its implications for the LHC", arXiv:1006.1901 [hep-th].

[37] A. Buchel, C. P. Herzog, I. R. Klebanov, L. A. Pando Zayas and A. A. Tseytlin, "Non-extremal gravity duals for fractional D3-branes on the conifold", JHEP 0104, 033 (2001) [arXiv:hep-th/0102105].

[38] P. Horava, E. Witten, "Eleven-dimensional supergravity on a manifold with boundary," Nucl. Phys. B475, 94-114 (1996) [hep-th/9603142].

[39] E. Silverstein, "Simple de Sitter Solutions", Phys. Rev. D 77, 106006 (2008) [arXiv:0712.1196 [hep-th]].

[40] S. S. Haque, G. Shiu, B. Underwood and T. Van Riet, "Minimal simple de Sitter solutions", Phys. Rev. D 79, 086005 (2009) [arXiv:0810.5328 [hep-th]]. 